Research Paper

\title{
The nucleosome binding protein 1 promotes the growth of gastric cancer cells
}

\author{
Lantao Liu1,\#, Zhifang Lang1,\#, Pengyu Wang1, Hongwei Wang2, Yanli Cao ${ }^{1}$, Xianghui Meng³, Jing Hu1, \\ Yukuan Feng, $1, \bowtie$ \\ 1. School of Basic Medicine, Mudanjiang Medical University, Mudanjiang 157011, P. R. China \\ 2. Department of Pathology, Hongqi Hospital Affiliated to Mudanjiang Medical University, Mudanjiang 157011, P. R. China \\ 3. Department of Dermatology, Hongqi Hospital Affiliated to Mudanjiang Medical University, Mudanjiang 157011, P. R. China \\ \# Contributed equally \\ $\triangle$ Corresponding author: Yukuan Feng, Professor, Key Laboratory of Heilongjiang Province for Cancer Prevention and Control, School of Basic Medicine, \\ Mudanjiang Medical University, Mudanjiang 157011, P. R. China. E-mail: fengyk@nsfc.gov.cn \\ (C) Ivyspring International Publisher. This is an open access article distributed under the terms of the Creative Commons Attribution (CC BY-NC) license \\ (https://creativecommons.org/licenses/by-nc/4.0/). See http://ivyspring.com/terms for full terms and conditions.
}

Received: 2018.08.16; Accepted: 2019.01.09; Published: 2019.01.29

\begin{abstract}
Nucleosome binding protein 1 (NSBPI) is identified as a new member of HMGN family and is abnormally overexpressed in a variety of tumors. However, it remains unclear whether NSBPI is overexpressed and promotes gastric cancer. In this study we employed RNAi mediated knockdown of NSBPI to investigate potential oncogenic role of NSBPI in gastric cancer. In BGC823 and SGC7901 gastric cancer cell lines, we showed that NSBPI knockdown decreased cell proliferation while increased apoptosis in vitro. Western blot analysis showed that NSBP1 knockdown decreased the levels of anti-apoptotic protein $\mathrm{Bcl}-2$ while increased the levels of pro-apoptotic protein Bax. In addition, NSBPI knockdown inhibited the growth and increased the apoptosis of SGC7901 cells xenografted in nude mice. In conclusion, this study provides the first evidence that NSBPI enhances the proliferation while inhibits the apoptosis of gastric cancer cells, and this is related to the regulation of the expression of apoptosis related proteins by NSBPI. These data suggest that NSBPI plays oncogenic role in gastric cancer.
\end{abstract}

Key words: NSBP1; Gastric cancer; apoptosis

\section{Introduction}

Despite recent advances in surgery and chemotherapy, gastric cancer remains a common tumor, especially in China which has the highest incidence of gastric cancer. Currently, gastric cancer is the third leading cause of cancer related death in China. Therefore, it is urgent to identify novel therapeutic targets for gastric cancer.

Nucleosome binding protein 1 (NSBP1, also called HMGN5) is identified as a new member of high mobility group N (HMGN) family, and it regulates a variety of processes such as DNA replication and repair, gene transcription, and epigenetic modifications $[1,2]$. NSBP1 has been reported to be abnormally overexpressed in several types of tumors such as breast cancer, bladder cancer, prostate cancer, glioma and osteosarcoma [3-7]. However, the expression and oncogenic role of NSBP1 in gastric cancer remain to be determined.

In this study we employed loss of function approach to knockdown NSBP1 in gastric cancer cell lines SGC7901 and BGC823 and evaluated the evaluated the effects on cell proliferation and apoptosis in vitro. In addition, we examined the growth and apoptosis rates of SGC7901 cells xenografted in nude mice after knockdown of NSBP1. Our results showed that NSBP1 knockdown inhibited the growth and promoted the apoptosis of gastric cancer cells both in vitro and in vivo, indicating oncogenic role of NSBP1 in gastric cancer. 


\section{Methods}

\section{Cell culture}

The normal gastric epithelial cell line GES-1 and human gastric cancer cell lines SGC7901 and BGC823 were purchased from Chinese Academy of Sciences (Shanghai, China), and maintained in RPMI1640 medium (Hyclone, Waltham, USA) supplemented with $10 \%$ fetal bovine serum (Hyclone), $100 \mathrm{U} / \mathrm{mL}$ penicillin and $100 \mathrm{mg} / \mathrm{mL}$ streptomycin at $37^{\circ} \mathrm{C}$ in a humid incubator with $5 \% \mathrm{CO}_{2}$.

\section{Lentivirus-mediated short hairpin RNA (shRNA) constructs}

The two shRNAs targeting human NSBP1 gene were synthesized by GenePharma (Shanghai, China). NSBP1 shRNA or control scramble shRNA was inserted into lentivirus vector $p S i c o R$ to make recombinant vectors pSicoR-NSBP1-shRNA and pSicoR-scramble-shRNA, which were co-transfected into HEK293 cells with lentiviral package system. The lentiviral particles were collected and titrated. SGC7901 and BGC823 cells were grown to 60-70\% confluence and then infected with appropriate amounts of lentivirus to knockdown NSBP1.

\section{Quantitative real-time PCR}

Total RNA was extracted from SGC7901 and BGC823 cells using Trizol reagent (Invitrogen) following the manufacturer's instruction and cDNA was synthesized using MMLV-reverse transcriptase (Promega, Madsion, WI, USA). Real-time PCR was performed on ABI Prism 7300 machine (Applied Biosystems, Foster City, CA, USA) with the following primers: NSBP1 5'-TCGGCTTTTTTTCTGCTGACT AA-3' and 5'-CTCTTTGGCTCCTGCCTCAT-3'; $\beta$-actin, 5'-GTGGACATCCGCAAAGAC3' and 5'-ATCAACGCAATGTGGGAAA-3'. Amplification conditions were as follows: initial denaturation for 3 min at $95^{\circ} \mathrm{C} ; 40 \mathrm{~s}$ at $94^{\circ} \mathrm{C}, 40 \mathrm{~s}$ at $58^{\circ} \mathrm{C}$ and $1 \mathrm{~min}$ at $72^{\circ} \mathrm{C}$ (30 cycles).

\section{Western blot analysis}

The proteins were extracted from SGC7901 and BGC823 cells using ice-cold RIPA buffer supplemented with protease and phosphatase inhibitors. Equal amounts of samples $(50 \mu \mathrm{g})$ were separated in 10\% SDS-polyacrylamide gels and then transferred onto PVDF membranes. After blocking in non-fat milk the membranes were incubated with primary antibodies against $\mathrm{Bax}, \mathrm{Bcl}-2$, and $\beta$-actin (all from Santa Cruz Biotech, Santa Cruz, CA, USA) at $4^{\circ} \mathrm{C}$ overnight, and then incubated with horseradish peroxidase conjugated secondary antibodies at $37^{\circ} \mathrm{C}$ for $1 \mathrm{~h}$. The blots were washed and exposed to enhanced chemiluminescence reagent.

\section{MTT assay}

SGC7901 and BGC823 cells were infected with lentivirus and then cultured in RPMI1640 medium. 24 $\mathrm{h}$ later, $15 \mathrm{ul}$ MTT solution was added into each well for incubation at $37^{\circ} \mathrm{C}$ for $4 \mathrm{~h}$. Next, $150 \mathrm{ul}$ DMSO was added into each well to dissolve the crystals, and the optical density at $450 \mathrm{~nm}$ was measured using a microplate reader.

\section{Flow cytometry analysis}

SGC7901 and BGC823 cells were infected with lentivirus and then cultured in RPMI 1640 medium. 24 $\mathrm{h}$ later, the cells were collected, fixed in $70 \%$ ethyl alcohol at $4^{\circ} \mathrm{C}$ overnight, then incubated with 10 $\mu \mathrm{g} / \mathrm{mL}$ RNAse at $37^{\circ} \mathrm{C}$ for $1 \mathrm{~h}$. Next, the cells were washed and resuspended in 400 Annexin $\mathrm{V}$ binding buffer (BD Biosciences, San Jose, CA, USA). The cells were then stained with $5 \mu$ l Annexin V FITC for 15 min at $4{ }^{\circ} \mathrm{C}$ in the dark followed by stained by propidium iodide (PI). The stained cells were then immediately subjected to flow cytometry analysis.

\section{Tumor xenograft assay}

All animal experiments were conducted following the Guidelines for the Care and Use of Laboratory Animals of Mudanjiang Medical University. Nude BALB/c mice (4-5 weeks old, weight around $20 \mathrm{~g}$ ) were kept in a pathogen-free facility. Uninfected SGC7901 cells or SGC7901 cells infected with NC shRNA or NSBP1 shRNA were harvested and cell suspensions $\left(1 \times 10^{7}\right.$ cells $)$ in $1 \mathrm{ml}$ RPMI1640 medium were injected into peritoneal cavity of nude mice. Tumor volume was calculated using the formula: the length $\times$ the width ${ }^{2} \times 0.5$.

\section{TUNEL assay}

Nude mice were sacrificed and tumor xenografts were dissected and fixed with $10 \%$ formalin for $4 \mathrm{~h}$, and then embedded and cut into sections. Apoptotic cells in tumor sections were analyzed using TUNEL kit (Roche Diagnostic, Indianapolis, IN, USA) following the manufacturer's instructions. Apoptotic cells showed brown nuclear staining, while hematoxylin counterstaining was used to detect all cells in the sections. Five fields were selected randomly from each section and the apoptotic rate was calculated by counting positively stained brown cells and total cells with the nuclei stained blue by hematoxylin.

\section{Statistical analysis}

Data were presented as mean \pm standard deviation and analyzed using SPSS 13.0 software (SPSS Inc., Chicago, IL, USA). P value $<0.05$ was judged as statistically significant. 


\section{Results}

\section{The expression of NSBPI is high in gastric cancer cells}

First we compared NSBP1 mRNA and protein expression levels in normal gastric epithelial cells and gastric cancer cells. By real-time PCR we found that NSBP1 mRNA level was significantly higher in BGC823 and SGC7901 cancer cells than that in GES-1 gastric epithelial cells (Fig. 1A). By Western blot analysis we found that NSBP1 protein level was higher in BGC823 and SGC7901 cancer cells than that in GES-1 cells (Fig. 1B, C). Collectively, these results demonstrate the overexpression of NSBP1 in gastric cancer cells.

\section{Knockdown of NSBP 1 inhibits gastric cancer cell proliferation}

To reveal the potential role of NSBP1 overexpression in gastric cancer, we made lentivirus shRNA constructs to knockdown NSBP1 in BGC823 and SGC7901 cells. By Western blot analysis we confirmed that two NSBP1 shRNAs but not scramble shRNA significantly reduced protein level of NSBP1 in BGC823 and SGC7901 cells (Fig. 2A, B). By MTT assay we found that knockdown of NSBP1 led to significant decrease in cell viability of BGC823 and SGC7901 cells (Fig. 2C).

\section{Knockdown of NSBP 1 enhances gastric cancer cell apoptosis}

To understand how NSBP1 promotes gastric cancer cell proliferation, we examined the apoptosis of BGC823 and SGC7901 cells depleted of NSBP1. By flow cytometry analysis we found that apoptotic cells were significantly more in the cells infected with NSBP1 shRNA compared to those infected with NC shRNA (Fig. 3A, B). By Western blot analysis we found that the level of anti-apoptotic Bcl-2 was lower while the level of pro-apoptotic Bax was higher in the cells infected with NSBP1 shRNA compared to those infected with NC shRNA (Fig. 3C).

\section{Knockdown of NSBP 1 kills gastric cancer xenografts}

To confirm our in vitro data, we injected SGC7901 cells depleted of NSBP1 into the peritoneal cavity of nude mice. After 4 weeks, we found that tumor volume of xenografts derived from cells depleted of NSBP1 decreased significantly compared to control groups (Fig. 4A, B).

Furthermore, by TUNEL assay we found that apoptotic rate of xenografts derived from SGC7901 cells depleted of NSBP1 decreased significantly compared to control groups (Fig. 4C, D). Collectively, these results indicate that NSBP1 inhibits cancer cell apoptosis to promote the tumorigenesis of gastric cancer.

\section{Discussion}

To our knowledge, this is the first report that NSBP1 was overexpressed in gastric cancer cells, in agreement with previous studies demonstrating that NSBP1 is overexpressed in numerous tumors and may play an oncogenic role [3-7]. As a new member of HMGN nuclear protein family, NSBP1 could modulate the structure and function of the chromatin and are crucially involved in the regulation of gene transcription as well as DNA replication and repair $[2,8]$.

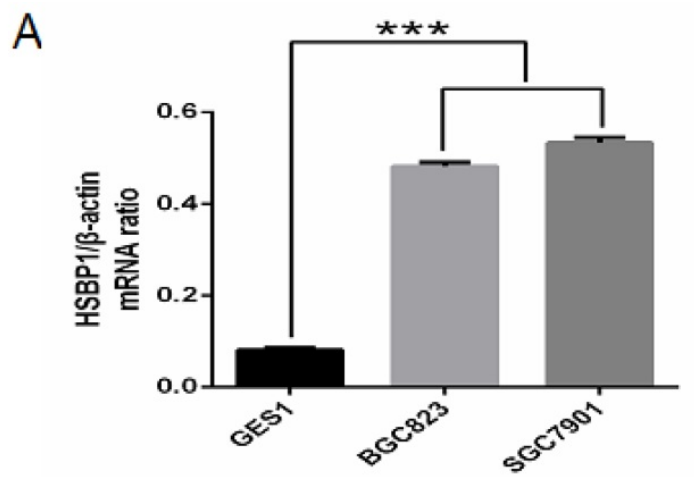

B
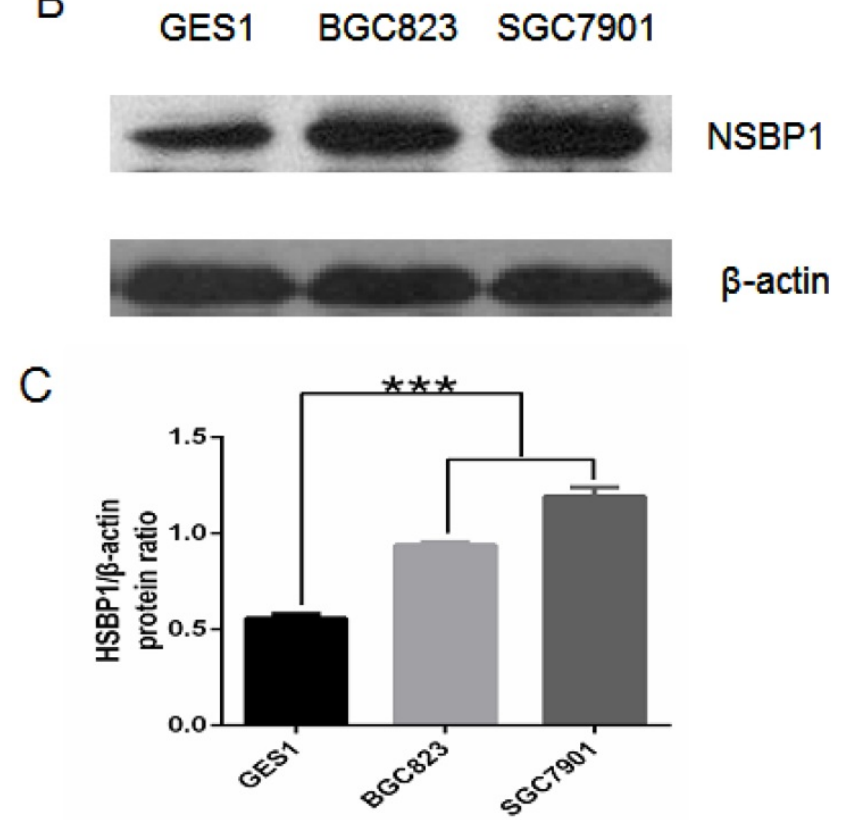

Fig. 1. Overexpression of NSBP1 in gastric cancer cells. A. Real-time PCR analysis of NSBP1 mRNA levels in GES-1, BGC823 and SGC7901 cells. Relative ratio of NSBP1 mRNA level was plotted against $\beta$-actin, which was internal control. $* * * P<0.001$. $B$. Western blot analysis of NSBP1 protein levels in GES-1, BGC823 and SGC7901 cells. Shown were representative blots from three experiments. C. Densitometry analysis of relative ratio of NSBP1 protein level $(n=3)$. $\beta$-actin was loading control. $* * * P<0.001$. 
A

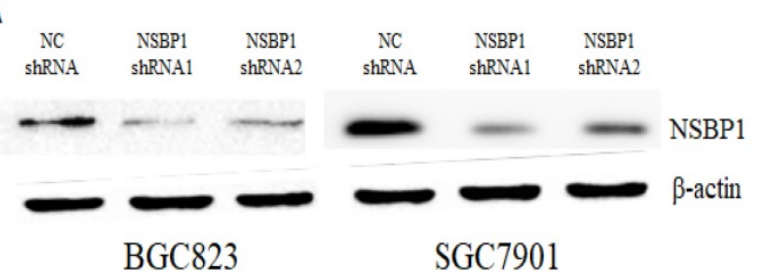

B

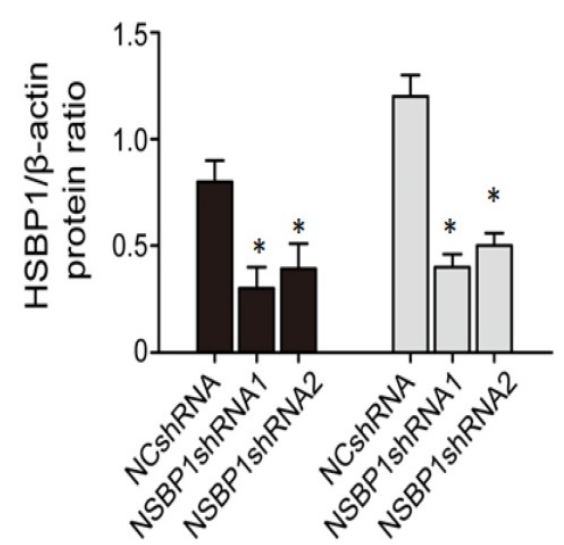

C

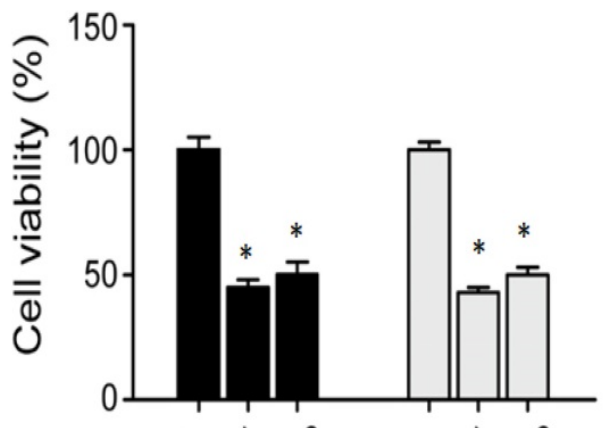

BGC823

$\square$ SGC7901

Fig. 2. Knockdown of NSBP1 inhibited gastric cancer cell viability. A. Western blot analysis of NSBP1 level in BGC823 and SGC7901 cells infected by NSBP1 shRNAs or scramble shRNA (negative control, NC shRNA). Shown were representative blots from three experiments. $\beta$-actin served as loading control. B. Densitometry analysis of NSBP1 level in BGC823 and SGC7901 cells infected by NSBP1 shRNAs or NC shRNA $(n=3) * P<0.05$ vs. NC shRNA. C. MTT assay of the viability of BGC823 and SGC7901 cells infected by NSBPI shRNAs or NC shRNA. Data represent mean \pm SD $(n=3)$. $* P<0.05$ vs. NC shRNA.

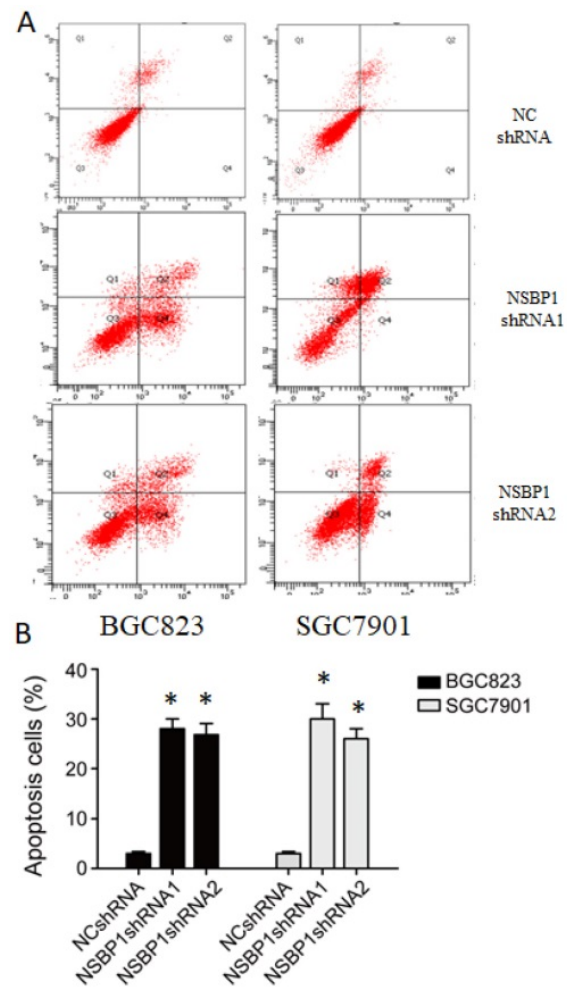

C $\begin{array}{clllll}\text { NC } & \text { NSBP1 } & \text { NSBP1 } & \text { NC } & \text { NSBP1 } & \text { NSBP1 } \\ \text { ShRNA } & \text { shRNA1 } & \text { ShRNA2 } & \text { shRNA } & \text { shRNA1 } & \text { shRNA2 }\end{array}$
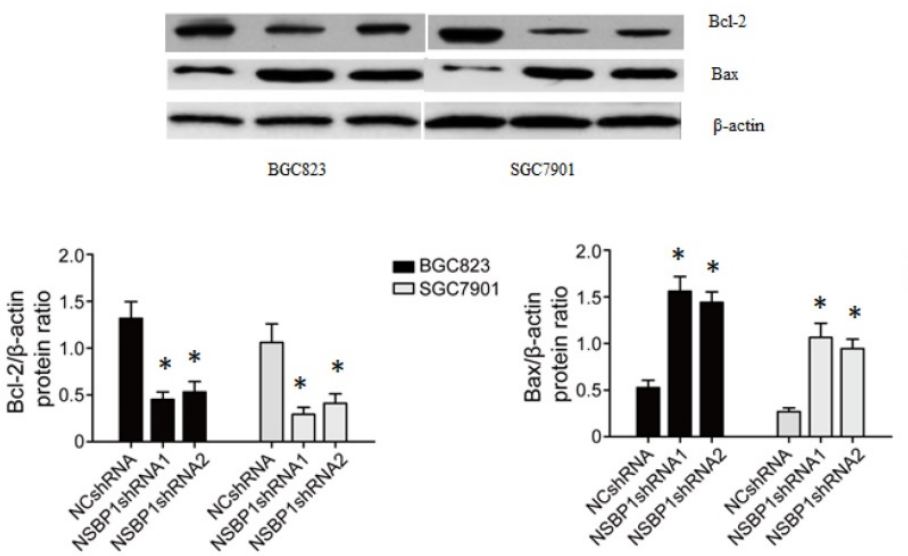

- BGC823 口SGC7901

Fig. 3. Knockdown of NSBPI enhanced gastric cancer cell apoptosis. A. Representative flow histograms on BGC823 and SGC7901 cells infected by NSBP1 shRNAs or NC shRNA. B. Quantitative analysis of apoptotic cells in BGC823 and SGC7901 cells infected by NSBPI shRNAs or NC shRNA. Data represent mean \pm SD ( $\mathrm{n}=3$ ). *P $<0.05$ vs. NC shRNA. C. Western blot analysis of Bcl-2 and Bax protein levels in BGC823 and SGC7901 cells infected by NSBP1 shRNAs or NC shRNA ( $\mathrm{n}=3$ ). ${ }^{*} \mathrm{P}<0.05$ vs. NC shRNA. 
A

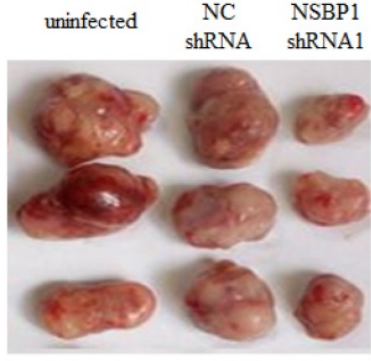

B

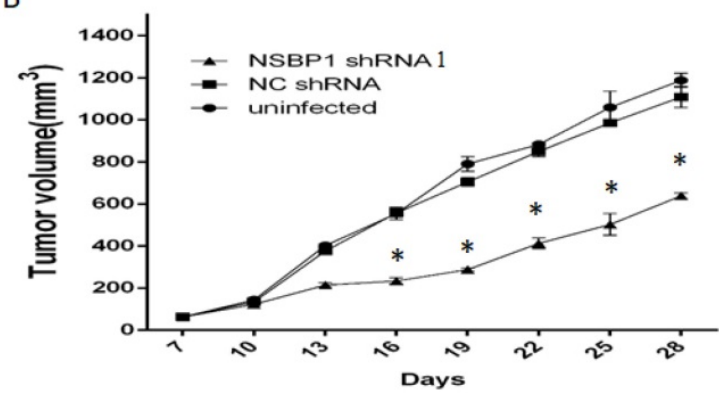

C

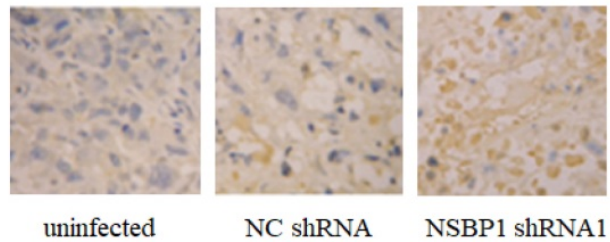

D

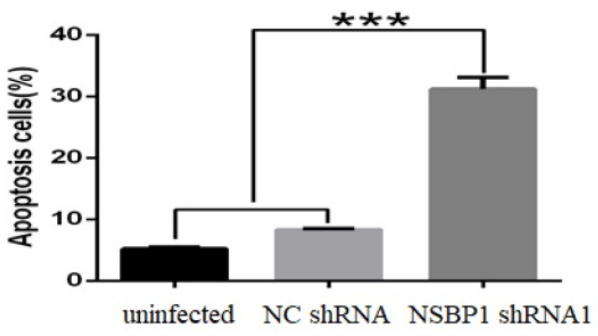

Fig. 4. Knockdown of NSBPI reduced in vivo tumorigenicity of gastric cancer cells. A. Typical tumors dissected from the xenografts in three groups of mice. B. Tumor growth curve of the xenografts in three groups. *P<0.05 vs. NC shRNA. C. Representative TUNEL staining of tumor sections. Apoptotic cells were stained brown and the nuclei were stained blue by counterstaining with hematoxylin. Original magnification $500 \times$. D. Quantitative analysis of the percentage of apoptotic cells $(n=3)$. $* * * P<0.001$.

Using a loss of function approach based on shRNA mediated knockdown of NSBP1 in two gastric cancer cell lines, we demonstrated that knockdown of NSBP1 led to reduced proliferation and increase apoptosis of gastric cancer cells, accompanied by the downregulation of anti-apoptotic protein $\mathrm{Bcl}-2$ and the upregulation of pro-apoptotic protein Bax. Collectively, these results indicate that NSBP1 may regulate the expression of apoptosis related proteins to inhibit the apoptosis and promote the proliferation of gastric cancer cells. A very recent study reported that HMGN5/NSBP1 promoted the proliferation and invasion of pancreatic ductal adenocarcinoma via the activation of Wnt/ $\beta$-catenin signaling [9]. Canonical Wnt/ $\beta$-catenin signaling is known to inhibit apoptosis and contribute to cancer resistance to chemoradiation therapy $[10,11]$. On the other hand, another recent study indicated that HMGN5/NSBP1 activated phosphatidylinositol 3-kinase/protein kinase $\mathrm{B} /$ the mammalian target of rapamycin pathway to promote renal cell carcinoma growth and metastasis [12]. Therefore, the activation of multiple signaling pathways by NSBP1 may contribute to its oncogenic role in a variety of cancer.

To confirm that NSBP1 is involved in the tumorigenesis of gastric cancer in vivo, we injected SGC7901 gastric cancer cells into nude mice to establish xenograft model. Based on this model we found that knockdown of NSBP1 inhibited tumor formation of the xenografts. Furthermore, we performed TUNEL assay on the xenografts and found that the apoptosis rate significantly increased in xenografts derived from SGC7901 cells depleted of NSBP1. These in vivo data complement the in vitro data and strongly suggest that NSBP1 promotes the tumorigenesis of gastric cancer via inhibiting apoptosis.

To understand how NSBP1 inhibits gastric cancer cell apoptosis, we demonstrated that knockdown of NSBP1 led to the upregulation of anti-apoptotic protein $\mathrm{Bcl}-2$ and the upregulation of pro-apoptotic protein Bax. However, it remains unclear how NSBP1 selectively upregulates anti-apoptotic proteins while downregulates pro-apoptotic proteins. In addition, other function of NSBP1 in gastric cancer such as tumor invasion and metastasis is largely unknown. For example, recent studies have shown that Akt/Girdin signaling is involved in the migration and invasion of human hepatocellular carcinoma [13,14]. Interestingly, NSBP1 increased human urothelial bladder cancer chemoresistance by upregulating PI3K/Akt signaling [15]. Further studies may focus on Akt signaling to explore the role of NSBP1 in gastric cancer invasion and metastasis.

In summary, this study provides the first evidence that NSBP1 promotes the proliferation while inhibits the apoptosis of gastric cancer cells, and this is related to the regulation of the expression of apoptosis related proteins by NSBP1. These data suggest that NSBP1 plays an oncogenic role in gastric cancer. Notably, recent studies have shown that lentivirus mediated shRNA against genes with potential oncogenic role provides a new avenue for developing 
effective therapy for a variety of cancers [16-20]. Therefore, it is promising to develop lentivirus mediated NSBP1 shRNA as a novel therapeutic approach for gastric cancer.

\section{Acknowledgments}

This study was supported by National Science Foundation of China (No. 81772498), Heilongjiang Province Science Key Funds (to Y.K.F.), Heilongjiang Province Science Foundation (No. H2018067), The Fundamental Research Funds for the Universities of Heilongjiang Province (No. 2017-KYYWFMY-0658, 0650, 0660 and 0661), The Postgraduate Innovative Research Fund of Mudanjiang Medical University (2017YJSCX-03MY).

\section{Competing Interests}

The authors have declared that no competing interest exists.

\section{References}

1. Rochman M, Postnikov Y, Correll S, Malicet C, Wincovitch S, Karpova TS, McNally JG, Wu X, Bubunenko NA, Grigoryev S, Bustin M. The interaction of NSBP1/HMGN5 with nucleosomes in euchromatin counteracts linker histone-mediated chromatin compaction and modulates transcription. Mol Cell. 2009;35:642-56

2. Rochman M, Malicet C, Bustin M. HMGN5/NSBP1: a new member of the HMGN protein family that affects chromatin structure and function. Biochim Biophys Acta. 2010; 1799:86-92

3. Wahafu W, He ZS, Zhang XY, Zhang CJ, Yao K, Hao H, Song G, He O, Li XS, Zhou LQ. The nucleosome binding protein NSBP1 is highly expressed in human bladder cancer and promotes the proliferation and invasion of bladder cancer cells. Tumour Biol. 2011;32:931-9.

4. Qu J, Yan R, Chen J, Xu T, Zhou J, Wang M, Chen C, Yan Y, Lu Y. HMGN5: a potential oncogene in gliomas. J Neurooncol. 2011;104:729-36.

5. Jiang N, Zhou LQ, Zhang XY. Downregulation of the nucleosome-binding protein 1 (NSBP1) gene can inhibit the in vitro and in vivo proliferation of prostate cancer cells. Asian J Androl. 2010;12:709-17.

6. Zhou X1, Yuan B, Yuan W, Wang C, Gao R, Wang J. The expression and clinical significance of high mobility group nucleosome binding domain 5 in human osteosarcoma. Tumour Biol. 2014:35:6539-47.

7. Weng M, Song F, Chen J, Wu J, Qin J, Jin T, Xu J. The high-mobility group nucleosome-binding domain 5 is highly expressed in breast cancer and promotes the proliferation and invasion of breast cancer cells. Tumour Biol. 2015;36:959-66.

8. Shirakawa H, Herrera JE, Bustin M, Postnikov Y. Targeting of high mobility group-14/-17 proteins in chromatin is independent of DNA sequence. J Biol Chem 2000; 275: 37937-44.

9. Zhao J, Wang Y, Wu X. HMGN5 promotes proliferation and invasion via the activation of Wnt/ $\beta$-catenin signaling pathway in pancreatic ductal adenocarcinoma. Oncol Lett. 2018;16:4013-4019.

10. Zhao Y, Tao L, Yi J, Song H, Chen L. The Role of Canonical Wnt Signaling in Regulating Radioresistance. Cell Physiol Biochem. 2018;48:419-432.

11. Tian H, Cong P, Qi R, Gao X, Liu X, Liu H. Shan F. Decreased invasion ability of hypotaurine synthesis deficient glioma cells was partially due to hypomethylation of Wnt5a promoter. Biocell 2017 41: 27-32

12. Wei $\mathrm{X}, \mathrm{Yu} \mathrm{L}$, Kong $\mathrm{X}$. miR-488 inhibits cell growth and metastasis in renal cell carcinoma by targeting HMGN5. Onco Targets Ther. 2018;11:2205-2216.

13. Ke Y, Bao T, Zhou Q, Wang Y, Ge J, Fu B, Wu X, Tang H, Shi Z, Lei X, Zhang C, Tan Y, Chen H, Guo Z, Wang L. Discs large homolog 5 decreases formation and function of invadopodia in human hepatocellular carcinoma via Girdin and Tks5. Int J Cancer 2017; 141:364-376.

14. Ke Y, Bao T, Wu X, Tang H, Wang Y, Ge J, Fu B, Meng X, Chen L, Zhang C, Tan Y, Chen H, Guo Z, Ni F, Lei X, Shi Z, Wei D, Wang L. Scutellarin suppresses migration and invasion of human hepatocellular carcinoma by inhibiting the STAT3/Girdin/Akt activity. Biochem Biophys Res Commun 2017; 483: 509-515.

15. Gan Y, He L, Yao K, Tan J, Zeng O, Dai Y, Liu J, Tang Y. Knockdown of HMGN5 increases the chemosensitivity of human urothelial bladder cancer cells to cisplatin by targeting PI3K/Akt signaling. Oncol Lett. 2017;14:6463-6470.
16. Li S, Wei Q, Li Q, Zhang B, Xiao Q. Down-regulating HIF-1a by lentivirus-mediated shRNA for therapy of triple negative breast cancer. Cancer Biol Ther. 2015;16:866-75.

17. Akhtar J, Wang Z, Yu C, Zhang ZP. Effectiveness of local injection of lentivirus-delivered stathmin1 and stathmin1 shRNA in human gastric cancer xenograft mouse. J Gastroenterol Hepatol. 2014;29:1685-91.

18. Feng Y, Tong X, Zhang B, Mao G, Huang H, Ma H. Effect of FAM196B in human lung adenocarcinoma. J Cancer. 2018;9:2451-2459.

19. Wang K, Xiao H, Zhang J, Zhu D. Synaptotagmin7 Is Overexpressed In Colorectal Cancer And Regulates Colorectal Cancer Cell Proliferation. J Cancer. 2018;9:2349-2356.

20. Tajima JY, Futamura M, Gaowa S, Mori R, Tanahashi T, Tanaka Y, Matsuhashi N, Takahashi T, Yamaguchi K, Miyazaki T, Yoshida K. Clinical Significance of Glycoprotein Non-metastatic B and Its Association with EGFR/HER2 in Gastrointestinal Cancer. J Cancer. 2018;9:358-366. 